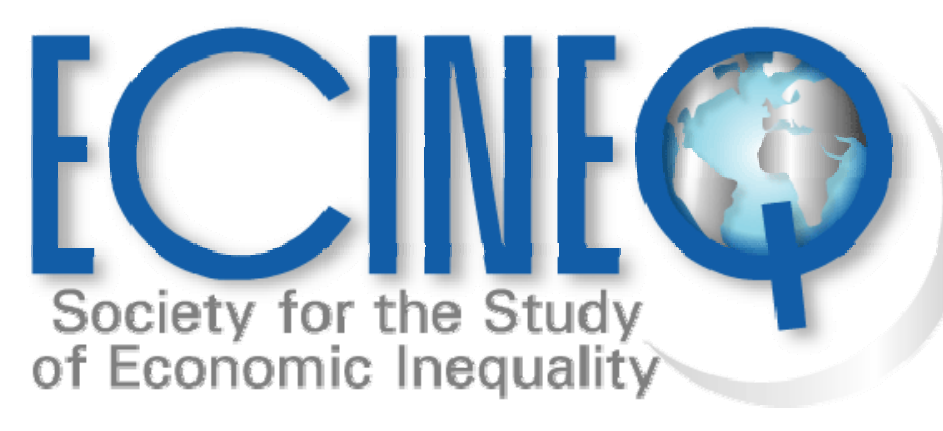

Working Paper Series

Reconsidering the Environmental Kuznets Curve hypothesis: the trade off between environment and welfare

Nicola Cantore 


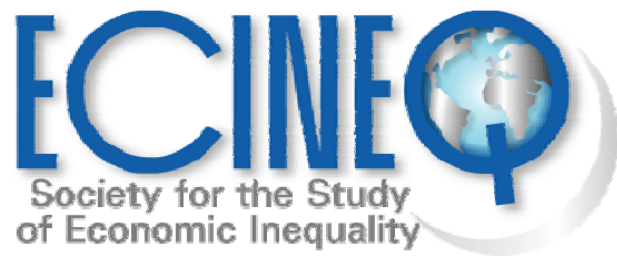

ECINEQ 2005-13

December 2005

www.ecineq.org

\title{
Reconsidering the Environmental Kuznets Curve hypothesis: the trade off between environment and welfare
}

\author{
Nicola Cantore* \\ University of York \\ Università Cattolica del Sacro Cuore, Milan
}

This version: December 2005

\begin{abstract}
Past climate change literature paid great attention to the welfare analysis of international agreements that stabilize emissions over time on the basis of the New Welfare Economics approach claiming "objective" measures of well-being and excluding interpersonal comparisons. In this paper, by using non New Welfare Economics approaches we show that the involvement of developing countries is not a desirable policy option. The implementation of a "Kyoto for ever" scenario including only developed regions could be recommended because improves both environment and welfare also if it does not generate a turning point in the relationship between income and pollution (PIR). The Environmental Kuznets Curve hypothesis (EKC) implies that a bell shaped PIR would induce policy-makers to pursue economic growth in order to overcome the air pollution issue. This normative prescription crucially focuses on the role played by the existence of a turning point in a context where only two sustainability dimensions are important: the economic and the environmental one. Our analysis shows that when we introduce a welfare analysis, policy implications based only on the turning point existence and consequently on the Environmental Kuznets Curve hypothesis could be misleading. In our study a "win-win" policy as the Kyoto Protocol is recommended because the existence of a turning point could be heavily paid in terms of welfare. However results are sensitive to the choice of the welfare measure..
\end{abstract}

Keywords: Environmental Kuznets Curve, climate change, welfare, income distribution..

JEL Classification: H0, H3, I3

*Contact details: nc141@york.ac.uk 


\section{Introduction}

Social choice in economics literature followed a long history, but it has seldom been connected to the environmental literature and in particular to the Environmental Kuznets Curve hypothesis. In the eighteenth century after the ideological incentive provided by the French Revolution and the birth of democracy Borda and Condorcet showed the problems connected in social choice by a democratic voting rule. 
Bentham introduced utilitarianism and the concept that the social welfare of a community can be accounted as the sum of utilities enjoyed by each individual. A cardinal approach was involved in the benthamian world and no distributional issues were considered. In the thirtieths the cardinal social welfare function was replaced by person's respective ordering of social states. Again interpersonal comparisons of utility gains and losses were neglected. The underlying criticism was that interpersonal comparisons of utility had no scientific basis. This change limited the informational basis on which to build the social choice.

In this new framework in the first half of the nineteenth century the New Welfare Economics (NEW) and the Pareto Criterion gave an important contribution in evaluating welfare changes in policies. The basic idea was that an alternative solution can be considered better if the change increases the utility of everyone, or at least, if it enhances the utility of at least one person and does not harm the interest o everyone. A further refinement of the Pareto criterion was the Kaldor-Hicks compensation test: if the gainers can compensate the losers and still better off (a potential Pareto improvement) then the policy is justified.

As pointed out by Gowdy (2005, pp.213):'The NWE vision was to establish appropriate criteria for evaluating distributional changes that do not involve value judgements". In this context Arrow (1950) by his impossibility theorem showed that under certain conditions it is impossible to reach a social choice. A great pessimism involved social choice theory and the possibility to reach social decisions involving divergent interests and concerns.

Recent developments have overcome the pessimism brought by the Arrow's impossibility theorem. In particular A. Sen (1999) shows that if we enlarge informational basis for social choice and we allow interpersonal comparisons among individuals the conclusions reached with the Arrow's theorem do not hold. Concretely when he writes about interpersonal comparisons he thinks to important issues as the income distribution, the poverty, the famine and various forms of inequality.

He rejects the traditional building blocks provided by the New Welfare Economics. Sen defends interpersonal comparisons of utilities based on mental states. Following Davidson (1986), he states that it is difficult to see how people can understand anything much about other people's minds and feelings without making some comparisons with their own minds and feelings.

$\mathrm{Ng}$ (1999) and others affirm that a Benthamian "hedonometer" could be obtained on the basis of scientific measures of well-being and a set of social, environmental, and economic indicators. Moreover he claims that it is not necessary to focus entirely on mental states. Utility can be considered as a matter of deprivation and "objectives" measures of need.

Starting from non-NWE approaches we have the opportunity to investigate two of the three dimensions mentioned in the Burtland report (1987) as necessary to investigate the sustainability issue: the economic and the social one. Our aim is to connect these issues with the last building block of the sustainability: the environmental one and in particular with the Environmental Kuznets curve analysis for a specific pollutant, $\mathrm{CO}_{2}$ emissions. The issue of the Environmental Kuznets curve has been largely debated over the last fifteen years. The normative prescription underlying the EKC hypothesis is extremely appealing: if we verify a bell shaped relationship between income and pollution, economic growth can be considered the best way to tackle the environmental issue. The crucial issue is the existence of a turning point in the relationship between income and pollution.

Past econometric evidence about the $\mathrm{EKC}$ existence for $\mathrm{CO}_{2}$ emissions showed that $\mathrm{EKC}$ is very unlikely to exist. The reason would lie in the global nature (Barbier, 1997) and in the intergenerational impacts (Ansategui and Escapa, 2002) of this pollutant. Specifically in this paper 
we will focus on emissions stabilizing policies aiming at reducing air pollution. Our contribution in this paper is twofold. We will investigate by the famous integrated climate change model RICE99 if international policies can generate a turning point in the relationship between income and pollution as postulated by the EKC hypothesis and their welfare effects on the basis of non-New Welfare Economics approaches.

EKC literature mainly consists of theoretical and econometric literature but little attention has been paid to numerical simulations and to the role played by emissions stabilizing policies. Moreover a great number of studies focus on the welfare effects of the Kyoto Protocol with or without the use of flexible mechanisms, but they are mainly based on New Welfare Economics approaches and equity implications are not analysed. Our work is aimed at filling these gaps. In climate change integrated assessment modelling a great number of studies focus on the impacts of emissions stabilizing international policies (Dagoumas et al. 2005, Jakeman et al. 2004, Buonanno et al. 2003). The basic finding is that the implementation of the Kyoto Protocol is costly for developed countries (in particular for USA), but the presence of an emission permits market, induced technical change or international trade can reduce the compliance costs.

A wide number of papers specifically deal with the impact in terms of welfare of international emissions stabilizing policies. Galeotti and Buchner (2003) survey the welfare effects of the Kyoto Protocol implementation. They find that for Annex I countries welfare losses are between $0.5 \%$ and $2 \%$, for non-AnnexI countries the effects are different for energy importers (gainers) and exporters (losers). Kemfert (2005), Edehnofer et al. (2005), Bernstein et al. (1999) show that the introduction of induced technical change reduces compliance welfare costs. Kuik and Gerlagh (2003) show that in a global perspective the Kyoto Protocol implementation is welfare reducing, but if we assume international trade and follow a Kaldor-Hicks Pareto Potential improvement criterion, the net gain among regions is positive. However all these studies do not include equity in their analysis. This is the core of our work.

Only a few attempts have been made in order to integrate economic and social issues with environmental issues in modelling literature. In particular only a few scholars link social and economic concerns with the climate change issue. Most of the previous studies focus on greenhouse gas emissions reductions so as to maximize human welfare on the basis of a neoclassical interpretation of justice (Nordhaus, 1991, Manne et al., 1995). The literature involving different welfare and equity issues in climate change is not wide.

Rose (1992) provides an interesting discussion of the economic, legal, and philosophical foundations of the equity criteria applicable to global warming policy. By a non-linear programming model, Rose and Stevens (1993) investigate the changes of welfare of permit allocations based on Sovereignty and Rawlsian equity criteria for 8 countries covering the spectrum of economic development levels.

Azar and Sterner (1996) show that the estimated marginal cost of $\mathrm{CO}_{2}$ emissions can be 50-100 times larger than the estimate by Nordhaus in his DICE model considering income distribution and a variable discount rate. Tol (2000) studies the implications in terms of equity in a context of welfare maximising greenhouse-gas emission reduction strategies. He analyses the impact of different principles of equity in emissions reduction strategies among regions. Bosello and Roson (1998) show that changes produced on an equity index by the imposition of emission constraints are not significantly higher than those obtained by the subsequent introduction of a market mechanism, and that the different market regimes which could be adopted have quite different distributional implications. Bosello, Buchner, Carraro and Raggi (2001) point out that even though more equitable 
burden sharing rules provide better incentives to sign and ratify a climate agreement than the burden-sharing rule implicit in the Kyoto Protocol, a stable global agreement cannot be achieved.

In our work we will show that the choice of the welfare measure in the analysis of emissions stabilizing policies is crucial and if we focus on non-NEW measures an environmental policy as the implementation of a "Kyoto forever" scenario is the best option.

On the basis of our analysis we will show that the existence of a turning point is not a reliable criterion to extract useful policy information. The existence of a turning point could be useful in terms of policy only if we work in a world composed of two dimensions: the economic and the environmental one. The reality is something more complex. If we add a third dimension (the welfare), policy recommendations based only on the turning point existence could be misleading.

The paper is organised as follows: in the section 2 we present the model and scenarios, in the section 3 results concerning EKC, in the section 4 we will present the definitions of welfare and the results, finally the conclusions.

\section{Model and scenarios}

\section{$\underline{\text { RICE99 }}$}

Nordhaus and Boyer (1999)'s RICE is the most recent version of a regional dynamic general equilibrium model for the study of the economic aspects of climate change (Nordhaus and Yang, 1996). The RICE model basically considers a single sector optimal growth model suitably extended to incorporate the interactions between economic activities and climate. There is one such model for each of the eight macro regions into which the world is divided: USA, Other High Income countries (OHI), OECD Europe (Europe), Eastern European countries (EE), Middle Income countries (MI), Lower Middle Income countries (LMI), China (CHN), and Low Income countries (LI). Within each region a central planner chooses the optimal paths of fixed investment and carbon energy input that maximizes the present value of per capita consumption. Nordhaus and Boyer's starting assumption is that a Social Planner optimally runs his/her own region, indexed by $n$, by maximizing the following discounted utility function:

$$
\underset{\{C(n, t)\}_{t=1}^{T}}{\operatorname{Max}} \sum_{t=1}^{T} \beta(t)^{t-1}(L(n, t) * \log (C(n, t) / L(n, t)))
$$

Where $C(n, t)$ stands for consumption, $\beta$ is the discount factor and $L(n, t)$ is the population level. The maximization process is subject to some constraints that capture the economic dynamics as well as the environmental ones.

The Resource Constraint of each region links consumption with net output $Y$ and with physical investments $I$. The following equation identifies the Resource Constraint ${ }^{1}$ :

$$
C(n, t)=Y(n, t)-I(n, t)
$$

\footnotetext{
${ }^{1}$ When we introduce an emissions permits market, equation (2) should also include the revenue (expenditure) for the sell (purchase) of permits.
} 
The gross value added obtained from the production process is described by the following equation:

$$
Q(n, t)=A(n, t)\left[K(n, t)^{\gamma} C E(n, t)^{\alpha n} L(n, t)^{(1-\gamma-\alpha n)}\right]-p_{e}(n, t) C E(n, t)
$$

Where $A(n, t)$ denotes the state of the technology, $K(n, t)$ is physical capital, $C E(n, t)$ is carbon energy, and $p_{e}(\mathrm{n}, \mathrm{t})$ is the price of carbon energy. Apart from $A(n, t)$ and $L(n, t)$, all the inputs of this value-added equation are endogenously determined. Note that the evolution of $A(n, t)$ accounts for productivity growth by production-enhancing technical change. In the model this index follows an exogenously determined increasing path over time.

There is a wedge $\Omega$ between gross and net output production due to climate alterations; this wedge is inversely related to and driven by the damage function $D(n, t)$ :

$$
\begin{aligned}
& Y(n, t)=\Omega(n, t) Q(n, t) \\
& \Omega(n, t)=1 / D(n, t) \\
& D(n, t)=1+\theta_{l, n} T(t)+\theta_{2, n} T(t)^{2}
\end{aligned}
$$

Where $D(n, t)$ is the environmental damage, $T(t)$ is the temperature and $\theta_{1, n}, \theta_{2, n}$ are regionalized parameters capturing the temperature impact.

The environmental damage is a key variable explaining how the model describes capital accumulation by including environmental resources. We refer to natural resources (intended as a flow) and not about environmental capital stocks, because the basic assumptions of this model are that there is an unlimited stock of natural resources and that carbon energy demand is always satisfied by supply. The scarcity is reflected only in the carbon price.

The green technological effect is described by

$$
E(n, t)=\varsigma(n, t) C E(n, t)
$$

Where $E(n, t)$ represents the level of emissions. Notice that the coefficient $\varsigma(n, t)$ in $(7)$ represents the emissions/carbon-energy ratio and captures the second form of technical change of the RICE99 model: emissions-reducing technical change. This index of carbon intensity is exogenously determined and follows a negative exponential path over time. In this way, Nordhaus and Boyer (1999) make the assumption of a gradual, costless improvement in green technology gained by the agents as time passes.

\section{The scenarios}

In our paper we will run four scenarios for RICE99 from 1995 to 2085:

- BAU (Business as usual)

- Kyoto scenario

- Global Kyoto scenario.

- Global Kyoto scenario 2. 
The BAU scenario implies no policy. In this scenario the economic growth has no limits for each region of the world. The non-cooperative game induces every social planner to improve economic development by an increasing level of emissions influencing the temperature and climate change. The Kyoto scenario implies a constraint on the emissions for each Annex I region (USA, OHI, Europe, EE) as imposed by the Kyoto Protocol over time. Moreover we include in the models a market of emissions trading ${ }^{2}$.

The Global Kyoto scenario implies an emission constraint for every region of the world including developing countries (MI, LMI, CHINA and LI). Given that the Kyoto Protocol does not include an emission constraint for developing countries we assume that the emissions constraint for each developing region is the level of emissions registered in the same region in 2015. Again we include in the models a market of emissions trading.

The Global Kyoto 2 scenario assumes a "Kyoto forever" constraint for developed countries as the Global Kyoto scenario. Since 2025 the emissions constraint over time for developing countries is $80 \%$ of the previous period BAU emissions. In this case we have no a fixed cap for poor regions, but the constraint is less strong.

Summarizing, with the Kyoto scenario we assume a lower emission constraint on a global scale but not caps on the emissions and consequently on the level of economic activity for developing countries. In the Global Kyoto scenario we assume a stronger emission constraint on a global scale but, at the same time, we introduce a strong limit for the economic growth in developing countries. The Global Kyoto2 scenario implies a softer emissions constraint for developing regions. The four scenarios are summarized in the table 1.

Table 1. Scenarios description

\begin{tabular}{|l|l|}
\hline Scenario & Description \\
\hline BAU & No policy. \\
\hline Kyoto & $\begin{array}{l}\text { Since 2015 Kyoto forever emission constraint for Annex I regions and market of } \\
\text { pollution permits. }\end{array}$ \\
\hline Global Kyoto & $\begin{array}{l}\text { Since 2015 Kyoto forever emissions constraint for Annex I regions. Developing } \\
\text { regions are obliged to maintain the level of emissions as in 2015. Market of pollution } \\
\text { permits. }\end{array}$ \\
\hline Global Kyoto 2 & $\begin{array}{l}\text { Since 2015 Kyoto forever emissions constraint for Annex I regions. Since 2025 } \\
\text { Developing regions are obliged to maintain } 80 \% \text { of the previous period BAU } \\
\text { emissions. Market of pollution permits. }\end{array}$ \\
\hline
\end{tabular}

\footnotetext{
${ }^{2}$ The emissions permits market is introduced by a non-cooperative Nash game in a dynamic setting, which yields an Open Loop Nash equilibrium This is a situation where in each region the planner maximizes its utility subject to the individual resource and capital constraints and the climate module for a given emission production of all the other players. Under the possibility of emission trading, the sequence whereby a Nash equilibrium is reached must be revised as follows. Each region maximizes its utility subject to the individual resource and capital constraints, now including the Kyoto constraint, and the climate module for a given optimal set of strategies of all the other players and a given price of permits $p(0)$ (in the first round this is set at an arbitrary level). When all regions have made their optimal choices, the overall net demand for permits is computed at the given price. If the sum of net demands in each period is approximately zero, a Nash equilibrium is obtained; otherwise the price is revised in proportion to the market unbalance and each region's decision process starts again
} 
We choose these scenarios because they appear as representative to answer the questions we posed in this paper.

Global warming has become a very worrying environmental issue and great debate developed towards emissions stabilizing policies. As we said above EKC literature showed that a bell shaped relationship between income and $\mathrm{CO}_{2}$ emissions is very unlikely to exist. In this paper first we will investigate if a BAU scenario generates a turning point. If a turning point is not reached and environmental policies are needed we will investigate the role played by international emissions stabilizing policies.

We will verify if an emissions constraint involving Annex I regions is a suitable policy option ("Kyoto forever" scenario) in order to reach a turning point or of it is necessary to impose an emissions constraint also for developing countries (Global Kyoto, Global Kyoto 2 scenario). We assume two possible emissions constraints for developing countries to check how the relationship between income and pollution evolves when we change the emissions constraint for poor regions.

The reader should notice that the Kyoto forever scenario involves also USA that have not yet ratified the Kyoto Protocol. We choose this scenario to analyse if USA ratification could be important in order to reach a turning point. Finally we will study the welfare impacts connected to each scenario. We will investigate if the turning point existence is compatible with welfare improvements in emissions stabilizing policies.

\section{The relationship between income and pollution: the results}

To analyse the relationship between income and air pollution we will focus on the relationship between global GDP per capita and emissions per capita The past literature has widely chosen a per capita version of the environmental indicator for empirical studies concerning EKC, Panayatou (1993), Selden and Song (1994).

The choice to work on a global scale lies on the discrepancy that can occur between EKC in individual countries and EKC in the world (Dinda, 2004). According to EKC hypothesis, the improvements in environmental quality are not attainable for the majority of the world population that has the standards of living substantially below the estimated turning points (Stern et al., 1996). The investigation of a world relationship between income and pollution is necessary. We consider the lapse of time 1995-2085.

As the reader can see from the Figure 1 the BAU scenario shows an increasing relationship between income per capita and emissions per capita. Precisely in RICE99 it is first decreasing and then continuously increasing and does not seem to reach a turning point. According to the EKC hypothesis the evidence showing an increasing relationship between income and pollution would suggest the need of environmental policies.

From the Figure 1 we can verify the environmental consequences deriving from three policies: the Kyoto Protocol and two versions of the Kyoto Protocol extended to developing countries. From our plots it is clear that the three policies decrease emissions, but only the Global Kyoto scenario can generate a decreasing relationship between income per capita and emissions per capita. In the Figure 1 the reader can verify in RICE99 a bell shaped relationship between income and pollution. Only if we extended the Kyoto Protocol to developing countries we could be more optimistic in terms of environmental sustainability. 
Figure 1. World GDP per capita (trillions of 1990 US\$/billions of population) vs world emissions per capita (Gigatons/ billions of population). Time period 1995-2085

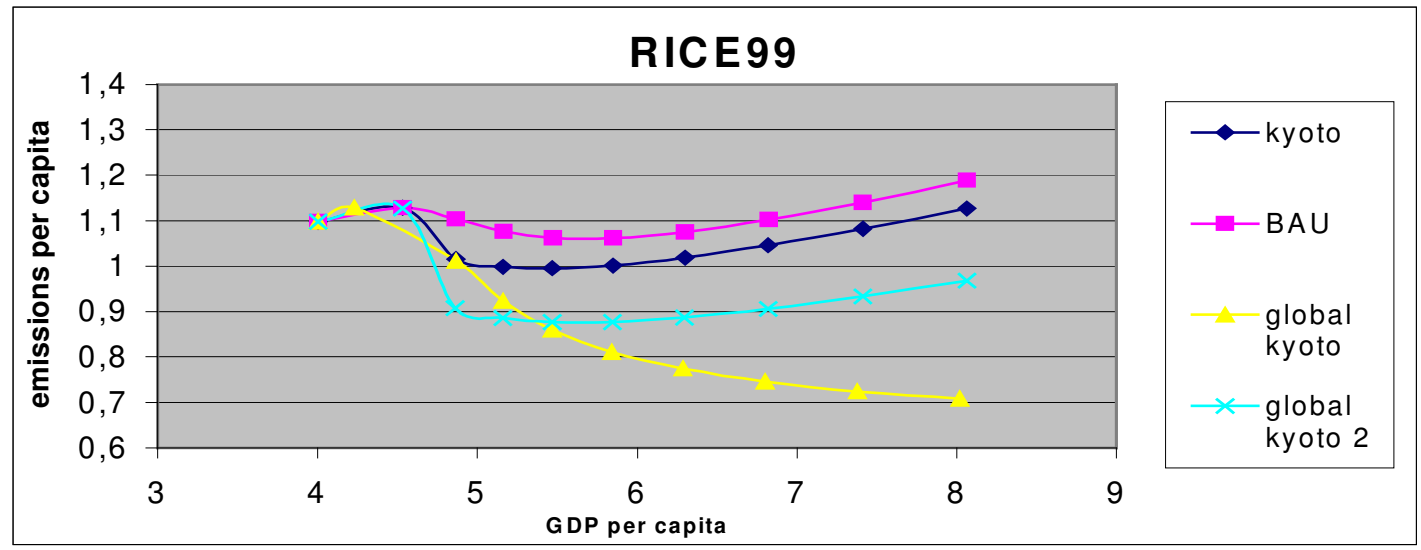

The Global Kyoto 2 scenario produces an increasing relationship between income and pollution because the emissions constraint for developing countries is less rigid than in Global Kyoto. This graph clearly explains the American administration argument rejecting the Kyoto Protocol ratification.

The American government position is to refuse the Kyoto Protocol ratification claiming that the climate change issue should be seriously tackled by involving developing countries. The existence of a turning point only when developing countries are strongly included in international agreements confirms the American objection. The problem is to verify what would be the consequences in terms of welfare

\section{Welfare. Definitions and results}

\subsection{Definitions}

As first indicator we will use a traditional measure of welfare that does not include equity criteria and represents a "metric" measure founded on economic values. Galeotti and Buchner (2003) implement a welfare analysis with RICE models by considering the present discounted value of consumption.

Specifically in this paper we will consider the present discounted value of world consumption over time. The measure of welfare is expressed by the following equation:

$$
\mathrm{W}(\mathrm{t})=\sum_{t=1}^{T} \beta(t)^{t-1} C(t)
$$

Where $\mathrm{C}(\mathrm{t})$ is the world consumption in each period $\mathrm{t}$ and $\beta$ is the discount factor. As the reader can easily see from this simple measure of welfare no consideration is given to how world consumption is distributed among regions over time. One of the aims of this paper is to investigate the link between a social welfare function based on equity and international climate change agreements. Past literature strongly highlighted the role of equity in influencing social choices. 
Atkinson's paper (1970) is the building block of this kind of literature. In his work, he points out the role of the income distribution in determining welfare rankings. Specifically he proposes a very popular index in order to provide a useful tool for social choice and provides criteria for the ranking of social states depending on the level of income $\mathrm{x}$ for different individuals $\mathrm{i}$. He considers a set of additive and separable Social Welfare Functions (SWFs) W as expressed in the following equation:

$$
\mathrm{W}=\sum \mathrm{U}_{\mathrm{i}}\left(\mathrm{x}_{\mathrm{i}}\right)
$$

Atkinson proves that assuming diminishing marginal utility of income, Lorenz curves ordering can rank alternative social states with the same average income. However, when Lorenz curves cross, it is always possible to find out different concave utility functions that can differently rank social states. Shorrocks (1983) extends Atkinson's formulation and introduces the concept of generalized Lorenz dominance. If the Lorenz curve of a distribution is $L(p)$ and the average income of the distribution is $\mu$, then the generalized Lorenz curve of this distribution is $\mu L(p)$. According to Shorrocks if the generalized Lorenz curve of one state lies above another, the social welfare corresponding to the first is said to be better than the latter ${ }^{3}$.

Both the Lorenz dominance and the generalized Lorenz dominance provide only partial ordering of the social welfare over time. In our paper for complete ordering we introduce a cardinal SWF that provides numerical values to all possible social states. As the Gini index $(G)$ is defined as twice the area between the Lorenz curve and the egalitarian line then $(1-G)$ is twice the area below the Lorenz curve. A cardinalization of the SWF can be obtained by finding the area below the generalized Lorenz curve. On the basis of an axioms set, Sen (1974) proposes a specific form of SWFs which is:

$$
\mathrm{W}(\mathrm{t})=\mu(\mathrm{t})(1-\mathrm{G}(\mathrm{t}))
$$

In our analysis $\mu$ is the average world consumption and $\mathrm{G}$ is the consumption concentration index among regions.

Another important social welfare function including the equity issue derives from the wide literature criticising GDP as the only factor influencing well-being. England (1998) surveys a number of quantitative measures of well-being which have been proposed as complements to or substitutes for GDP. Quoting England the consequent issues can be summarized as:

- The need to properly specify the distinction between intermediate and gross final output;

- The need to account or asset depreciation in a comprehensive manner;

- The need to divide net final output between consumption and capital accumulation on a reasonable basis;

- The need to take into account of the welfare implications of various forms of social inequality.

About the last point, England points out that the UNDP uses real per capita GDP as one component of the Human Development Index ${ }^{4}$ and since 1991 an Atkinson-style utility function has been used to transform a nation's income level into a measure of national social welfare $\mathrm{U}$ for each region n:

$$
\mathrm{U}(\mathrm{n}, \mathrm{t})=\frac{y(n, t)^{(1-\varepsilon)}}{(1-\varepsilon)}
$$

\footnotetext{
${ }^{3}$ When generalised Lorenz curves cross other theoretical contributions provide tools to rank alternative social states (see Atkinson A.B. and Bourgignon F. (1987), Dardanoni V. and Lambert P. (1988)).

${ }^{4}$ The Human Development Index also focuses on longevity and access to education.
} 
Where y is a country's per capita income, $\mathrm{y}^{*}$ is global per capita income, $\varepsilon=\alpha /(\alpha+1)$ with $\alpha$ nonnegative integer and $\alpha y^{*}<y<(\alpha+1) y^{*}$. For a nation with a per capita GDP below the global average, $\varepsilon=0$ and $\mathrm{U}(\mathrm{n}, \mathrm{t})=\mathrm{y}(\mathrm{n}, \mathrm{t})$.

The UNDP social welfare function has been thought to transform nations' income levels, but with RICE99 we transform the consumption per capita for each region. Given U(n,t) the utility function of each region n, we only assume that the global social welfare function is an additive and separable function of the utilities enjoyed by each region.

The global SWF function will be:

$$
\mathrm{W}(\mathrm{t})=\sum_{\mathrm{n}} \mathrm{U}(\mathrm{n}, \mathrm{t}) \text {. and } \mathrm{U}(\mathrm{n}, \mathrm{t})=\frac{y(n, t)^{(1-\varepsilon)}}{(1-\varepsilon)} \text { as in }(11)
$$

Besides the equations (8), (10) and (12) we will utilise another social welfare function to assess welfare in different environmental policies scenarios. As we previously said, Sen (1999) criticizes the NEW approach and considers as crucial in the social choice process not only the interpersonal comparisons based on deprivation, but also those based on mental states. Sen is not the only economist who shares this vision.

Gowdy (2005 pp.218) states:” It has been conclusively demonstrated that welfare changes cannot be evaluated without making interpersonal comparisons of utility. Yet most economists are reluctant to accept "subjective" measures of well-being... The increasingly high level of psychology has helped to make the idea of measurable utility acceptable to economists". Kahneman et al. (1997) claim that subjective and hedonic experience can be observed and measured.

Though in a wide share of environmental models the utility function is characterized by psychological assumptions about the human behaviour (monotonicity, non-satiation), economists generally seek monetary measures of well-being to extract useful welfare implications. As wellbeing indicator in RICE99 we will consider again an additive and separable social welfare function depending on the utility function of each region as expressed in the equation (1). Therefore

$$
\mathrm{W}(\mathrm{t})=\sum_{\mathrm{n}} \mathrm{U}(\mathrm{n}, \mathrm{t}) \text { where } \mathrm{U}(\mathrm{n}, \mathrm{t})=\sum_{t=1}^{T} \beta(t)^{t-1}(L(n, t) * \log (C(n, t) / L(n, t)))
$$

By this social welfare function a transfer of one unity of consumption per capita from the richest countries to developing regions is welfare improving. This notion of welfare considers a discounted flow of consumption until the period t. The social welfare function in (13) is similar to the equation in (12).

The main differences are:

- The presence of the discount factor $\beta$ decreasing the weight attributed to the consumption in the future periods.

- The constant elasticity of the marginal utility of consumption for each region. The logarithmic form of the utility function is excluded in (11) given that $\varepsilon=\alpha /(\alpha+1)$ and $\varepsilon$ can never be equal to one.

- The argument of this welfare function concerns discounted sum of consumption over time and not consumption in each period. This is a "stock" more than "flow" measure of welfare. 
The application of these welfare functions in RICE99 implies the existence of a World Social Planner (WSP) aimed at assessing the results deriving from different scenarios and from the maximization process of each region. In applying the Sen's social welfare function (10) the WSP considers data deriving from RICE99 as representing the future real world and maximizes an additive and separable SWF depending on the utility function of each individual as in (9). Being the RICE99 model based on a regional framework, in our analysis we are implicitly assuming that the consumption in each region is equally distributed among people.

Equations (8), (10), (12) and (13) will be used to assess international emissions reducing policies. We are interested to evaluate if different notions of social welfare function based on different approaches drive to the same conclusions in terms of environmental policy. The choice of more welfare measures is a useful exercise to verify the robustness of our results.

Our aim is twofold:

- To compare traditional measures of welfare with measures deriving from non-New Welfare Economics approaches in different scenarios;

- To extract useful policy insight from the observation of welfare paths and our previous investigation of emissions paths associated to our set of scenarios.

The results are presented in the next section.

\section{$\underline{4.2 \text { Results }}$}

First we show the results relative to the traditional measure of welfare. As the reader can easily see from the table 2 the BAU scenario is the best over time because shows the highest level of discounted consumption per capita.

The result derives from an easy intuition. Emissions constraints are costly in terms of consumption. In the Kyoto scenario developed countries must accomplish the binding emission constraint and are committed to reduce the level of economic activity and consequently the level of consumption. This induces a lower level of global consumption per capita.

When we analyse the GK and GK2 scenarios, welfare losses are higher than in a Kyoto scenario. Emissions constraints strongly penalise growth and consumption in poor regions. The world consumption per capita is lower because both developed and developing countries are subject to an emissions constraint and are constrained to decrease the level of the economic activity.

Results are quite different when we include equity criteria in our analysis. In the table 3 we show the results concerning the Sen's Social Welfare function. The reader can see that since 2015 a "Kyoto forever scenario" always shows welfare gains. Scenarios involving also developing countries are the worst in terms of welfare over time.

These results can be easily understood reading tables showing average consumption and the value associated to the GINI coefficient for each scenario in the table 4. Over time the best scenario in terms of consumption per capita is the BAU scenario (except in 2085 where less environmental damage generates a higher consumption per capita in the Kyoto scenario). However the Kyoto scenario shows the best results in terms of equity. An emissions constraint involving developed countries decreases growth in rich countries and consequently growth in the world but increases equity over time. The Kyoto scenario shows a trade off: less average consumption but more equity than a BAU scenario. By the Sen's social welfare function the trade off is solved in favour of the Kyoto Protocol implementation. 
About the scenarios involving developing countries we find less average consumption and less equity than in a BAU or in a Kyoto scenario. Emissions constraints imposed to poor regions reduce world consumption growth and generate more inequality. There are two main differences between the GK and the GK2 scenario results:

- In the initial periods the emissions constraint is more stringent in the GK2 scenario for developing countries and consequently the welfare loss is higher. In 2015 in the GK scenario poor regions are constrained by their level of BAU emissions and as consequence are not committed to decrease their level of economic activity;

- Over time the fixed emissions cap in the GK scenario is much more stringent than the increasing one in the GK2 scenario. Whereas in the GK scenario we register raising welfare losses, in the GK2 scenario we observe an opposite trend.

Only a Kyoto scenario always shows welfare gains. Results suggest that an emissions stabilizing policy involving only developed countries is strongly recommendable.

Table 2. A traditional measure of welfare: \% differences of environmental policies vs BAU scenario. 1995-2085.

\begin{tabular}{|c|c|c|c|}
\hline & Kyoto vs BAU & GK vs BAU & GK2 vs BAU \\
\hline 1995 & 0,000 & 0,000 & 0,000 \\
\hline 2005 & 0,000 & 0,000 & 0,000 \\
\hline 2015 & $-0,001$ & 0,000 & $-0,001$ \\
\hline 2025 & $-0,002$ & $-0,002$ & $-0,005$ \\
\hline 2035 & $-0,003$ & $-0,004$ & $-0,008$ \\
\hline 2045 & $-0,003$ & $-0,006$ & $-0,010$ \\
\hline 2055 & $-0,004$ & $-0,009$ & $-0,011$ \\
\hline 2065 & $-0,004$ & $-0,012$ & $-0,012$ \\
\hline 2075 & $-0,004$ & $-0,015$ & $-0,013$ \\
\hline 2085 & $-0,004$ & $-0,018$ & $-0,013$ \\
\hline
\end{tabular}

Table 3. The Sen's Social Welfare function. Time period: $1995-2085$. $\%$ differences of environmental policies vs BAU scenario.

\begin{tabular}{|c|c|c|c|}
\hline & Kyoto vs BAU & GK vs BAU & GK2 vs BAU \\
\hline 1995 & 0,004 & 0,005 & 0,004 \\
\hline 2005 & 0,007 & 0,011 & 0,009 \\
\hline 2015 & 0,018 & 0,019 & 0,023 \\
\hline 2025 & 0,014 & $-0,067$ & $-0,336$ \\
\hline 2035 & 0,023 & $-0,204$ & $-0,324$ \\
\hline 2045 & 0,040 & $-0,370$ & $-0,283$ \\
\hline 2055 & 0,058 & $-0,567$ & $-0,236$ \\
\hline 2065 & 0,071 & $-0,795$ & $-0,187$ \\
\hline 2075 & 0,080 & $-1,045$ & $-0,133$ \\
\hline 2085 & 0,088 & $-1,308$ & $-0,071$ \\
\hline
\end{tabular}


Table 4. The Sen's Social Welfare function decomposition by per capita consumption (PCC, trillions of 1990 US\$/billions of population) and the Gini index. Time period: 1995 - 2085.

\begin{tabular}{|c|c|c|c|c|}
\hline & & BAU & & \\
\hline & PCC & Gini & 1-GINI & Utility \\
\hline 1995 & 2,979 & 0,643 & 0,357 & 1,062 \\
\hline 2005 & 3,432 & 0,610 & 0,390 & 1,338 \\
\hline 2015 & 3,710 & 0,582 & 0,417 & 1,548 \\
\hline 2025 & 3,948 & 0,556 & 0,443 & 1,751 \\
\hline 2035 & 4,180 & 0,530 & 0,470 & 1,963 \\
\hline 2045 & 4,463 & 0,506 & 0,493 & 2,204 \\
\hline 2055 & 4,800 & 0,483 & 0,516 & 2,478 \\
\hline 2065 & 5,190 & 0,463 & 0,537 & 2,787 \\
\hline 2075 & 5,630 & 0,444 & 0,556 & 3,131 \\
\hline 2085 & 6,122 & 0,426 & 0,574 & 3,512 \\
\hline
\end{tabular}

\begin{tabular}{|c|c|c|c|c|}
\hline & & Kyoto & & \\
\hline & PCC & Gini & 1-GINI & Utility \\
\hline 1995 & 2,979 & 0,643 & 0,357 & 1,062 \\
\hline 2005 & 3,432 & 0,610 & 0,390 & 1,338 \\
\hline 2015 & 3,707 & 0,582 & 0,418 & 1,548 \\
\hline 2025 & 3,945 & 0,556 & 0,444 & 1,751 \\
\hline 2035 & 4,177 & 0,530 & 0,470 & 1,963 \\
\hline 2045 & 4,461 & 0,505 & 0,494 & 2,205 \\
\hline 2055 & 4,799 & 0,483 & 0,517 & 2,480 \\
\hline 2065 & 5,189 & 0,462 & 0,537 & 2,789 \\
\hline 2075 & 5,630 & 0,443 & 0,556 & 3,133 \\
\hline 2085 & 6,124 & 0,426 & 0,574 & 3,515 \\
\hline
\end{tabular}

\begin{tabular}{|c|c|c|c|c|}
\hline & & GK & & \\
\hline & PCC & GINI & 1-GINI & Utility \\
\hline 1995 & 2,979 & 0,643 & 0,357 & 1,062 \\
\hline 2005 & 3,432 & 0,610 & 0,390 & 1,338 \\
\hline 2015 & 3,709 & 0,583 & 0,417 & 1,548 \\
\hline 2025 & 3,945 & 0,556 & 0,444 & 1,750 \\
\hline 2035 & 4,173 & 0,531 & 0,469 & 1,959 \\
\hline 2045 & 4,452 & 0,507 & 0,493 & 2,196 \\
\hline 2055 & 4,784 & 0,485 & 0,515 & 2,464 \\
\hline 2065 & 5,166 & 0,465 & 0,535 & 2,765 \\
\hline 2075 & 5,598 & 0,447 & 0,553 & 3,098 \\
\hline 2085 & 6,080 & 0,430 & 0,570 & 3,466 \\
\hline
\end{tabular}

\begin{tabular}{|c|c|c|c|c|}
\hline & & GK2 & & \\
\hline & PCC & GINI & 1-GINI & Utility \\
\hline 1995 & 2,979 & 0,643 & 0,357 & 1,062 \\
\hline 2005 & 3,432 & 0,610 & 0,390 & 1,338 \\
\hline 2015 & 3,707 & 0,582 & 0,418 & 1,548 \\
\hline 2025 & 3,939 & 0,557 & 0,443 & 1,745 \\
\hline 2035 & 4,170 & 0,531 & 0,469 & 1,957 \\
\hline 2045 & 4,454 & 0,507 & 0,493 & 2,198 \\
\hline 2055 & 4,792 & 0,484 & 0,516 & 2,472 \\
\hline 2065 & 5,183 & 0,463 & 0,537 & 2,781 \\
\hline 2075 & 5,625 & 0,444 & 0,556 & 3,126 \\
\hline 2085 & 6,121 & 0,427 & 0,573 & 3,509 \\
\hline
\end{tabular}


From the table 5 if we consider the UNDP social welfare function the results are quite similar. In the equation (12) the Gini concentration index does not play a role, but the consumption per capita of each region is weighted according to equity criteria. In the "Kyoto forever" scenario three effects influence welfare:

- Developed countries reduce consumption because they are subject to an emissions constraint;

- Eastern Europe Countries gain from the implementation of an emissions permits market because they are the most important permits sellers among Annex I regions;

- The Kyoto Protocol implementation generates less emissions and lower environmental damages affecting also developing countries (see equations $4,5,6$ ).

Given the framework of equation (12) the decrease of consumption per capita for developed countries that are subject to an emissions constraint has a lower impact than the gains for developing regions and Eastern Europe Countries deriving respectively from a lower environmental damage and the sell of permits in the emissions market. For this reason the "Kyoto forever" scenario provides the best welfare performances.

Again GK and GK2 show high levels of welfare losses deriving from the high consumption per capita reductions in developing countries generated by the emissions constraint. However as in the previous case the welfare loss seems to increase over time with a more severe emissions constraint as in the GK scenario.

Table 5 The UNDP Social Welfare function. Time period: 1995 - 2085. \% differences of environmental policies vs BAU scenario.

\begin{tabular}{|c|c|c|c|}
\hline & Kyoto vs BAU & GK vs BAU & GK2 vs BAU \\
\hline 1995 & 0,002 & 0,002 & 0,002 \\
\hline 2005 & 0,004 & 0,004 & 0,004 \\
\hline 2015 & 0,021 & 0,002 & 0,022 \\
\hline 2025 & 0,020 & $-0,004$ & $-0,027$ \\
\hline 2035 & 0,018 & $-0,014$ & $-0,029$ \\
\hline 2045 & 0,017 & $-0,028$ & $-0,029$ \\
\hline 2055 & 0,017 & $-0,048$ & $-0,027$ \\
\hline 2065 & 0,018 & $-0,077$ & $-0,024$ \\
\hline 2075 & 0,019 & $-0,114$ & $-0,019$ \\
\hline 2085 & 0,019 & $-0,162$ & $-0,012$ \\
\hline
\end{tabular}

Finally we can comment social welfare deriving from the "subjective" definition of welfare. From the table 6 the reader can notice that again the "Kyoto forever" scenario is welfare improving over time and the worst scenarios are those involving developing countries (GK and GK2). 
Table 6 A "subjective" Social Welfare function. Time period: 1995 - 2085. \% differences of environmental policies vs BAU scenario.

\begin{tabular}{|c|c|c|c|}
\hline & Kyoto vs BAU & GK vs BAU & GK2 vs BAU \\
\hline 1995 & 0,000 & 0,001 & 0,001 \\
\hline 2005 & 0,001 & 0,002 & 0,001 \\
\hline 2015 & 0,002 & 0,003 & 0,002 \\
\hline 2025 & 0,002 & 0,000 & $-0,012$ \\
\hline 2035 & 0,002 & $-0,006$ & $-0,019$ \\
\hline 2045 & 0,003 & $-0,013$ & $-0,024$ \\
\hline 2055 & 0,003 & $-0,021$ & $-0,026$ \\
\hline 2065 & 0,004 & $-0,029$ & $-0,027$ \\
\hline 2075 & 0,004 & $-0,038$ & $-0,027$ \\
\hline 2085 & 0,004 & $-0,046$ & $-0,027$ \\
\hline
\end{tabular}

The utility function of each region in the "subjective" welfare index is less equalitarian oriented. Whereas in the subjective index the logarithmic form of the utility function is the same for all the regions, in the UNDP index the elasticity of the marginal utility of income is higher for rich regions and the "weight" for developing regions is higher. A less equalitarian utility function, the presence of the discount rate and the fact that we sum weighted sums of consumption per capita over time does not qualitatively change our results when compared to those relative to the UNDP index.

The "Kyoto forever" scenario is welfare improving, emissions stabilizing policies involving also developing countries are welfare reducing. However if the emissions constraint for developing countries is less severe welfare losses are lower. In this case we cannot register a decreasing level of welfare loss for the GK2 scenario. Our "subjective" index is a "stock" measure of welfare depending on the cumulated sum of consumption flows over time. The decreasing path of welfare losses that we found in "flow" expressions of welfare is reflected in an increasing path of welfare loss with this "stock" index.

These results clearly explain the reluctance of the European Union about the involvement of developing countries in an international emissions stabilizing agreement: it is preferable to obtain a small environmental improvement without negative welfare implications as the Kyoto Protocol rather than policies aimed at obtaining a turning point in the income/pollution relationship paying an high cost in terms of well-being as the GK scenario.

Of course the American and European positions about the Kyoto Protocol ratification cannot be interpreted only on the basis of "altruistic" considerations. Past literature widely showed that the soft position of the European Union is mainly determined by the lower abatement costs than USA in Kyoto compliance costs (Nordhaus and Boyer 1999).

However our aim is to underline a crucial policy implication: when we introduce a third dimension in the Environmental Kuznets Curve analysis, namely the welfare in our paper, the turning point existence is not crucial to extract useful environmental policy implications. The Kyoto Protocol reduces emissions if compared with a BAU scenario and is welfare improving on the basis of measures including economic and equity components. The implementation of "win-win" policies as the "Kyoto forever" scenario that do not reach a turning point in the relationship between income and pollution can be more recommendable than strong welfare reducing policies as the GK scenario. 


\section{Conclusions}

In this paper we investigated four scenarios involving different hypothesis about regions' participation to international emissions stabilizing programs. Following a multidimensional approach concerning sustainability we analysed the economic, social and environmental impact of each scenario. Specifically we studied the relationship between income and pollution and by nonNew Welfare Economics approaches the level of welfare associated to each scenario.

We found first that the only scenario implying a welfare and environmental improvement is the "Kyoto forever" one that does not generate a turning point in the PIR. Emissions constraints for developing countries are strongly welfare reducing. From this finding we can derive two important policy implications.

First, the choice of the welfare measure to analyse policies is crucial. As we saw in our paper when we consider measures of welfare including equity criteria the implementation of the Kyoto Protocol is more preferable than a no-policy scenario. We obtain the opposite result with a traditional measure based on consumption. The welfare measure is a tool that must be cautiously managed by the policy maker. The "right" welfare measure will be chosen on the basis of the policy targets. A drawback of our analysis is that we worked on the basis of "global" measures of welfare. The underlying hypothesis is that a World Agency is interested to account the world level of welfare in different scenarios and to choose the best option on the basis of a global interest. This could be considered as unrealistic. Game theory explains that in the global warming issue the most realistic view is to consider maximizing agents that behave on the basis of their own self-interests. Recent policies about debt cancellation and the Kyoto Protocol ratification of the most part of the Annex I countries are useful examples showing that in future years ethical and social justice could drive international policies in a context of global sustainability. This paper captures this alternative view.

Second we analysed the role played by the existence of a turning point in the relationship between income and pollution as criterion to determine environmental policies. EKC hypothesis postulates that if we observe a bell shaped relationship between income and pollution, fostering economic growth is the best policy to tackle the pollution issue. The existence of a turning point is considered as the crucial factor in order to set an environmental policy and past theoretical and empirical literature mainly focussed on the shape of the relationship between income and pollution. In this paper we criticize this approach. We showed that the existence of a turning point could not be a suitable social choice criterion. The Environmental Kuznets Curve solves the traditional trade off between income and environment, but could be strongly welfare reducing. When we introduce other policy targets other than environment and income, the EKC could not be a suitable tool to suggest policies. The empirical observation of the relationship between income and pollution is a useful evidence for the policy maker in order to set environmental policies, but cannot considered as unique criterion. The seeking of "win-win" policies generating economic, social and environmental improvements also if they do not generate a turning point seems to be the best tool for social choice.

However when we cannot find "win-win" policies the social choice theory should be the tool to solve the trade offs. All the literature concerning equity since the Atkinson's findings widely increased the opportunity to reach a social choice managing the trade off between economic and social variables. Further effort should be addressed to build social welfare functions to manage trade offs concerning all dimensions of sustainability: the economic, the environmental and the social one. The policy prescription deriving from the Environmental Kuznets Curve hypothesis seems not be sufficient to derive useful insights when more policy targets are involved. 
Further research, rather than focussing on the existence of a turning point in the relationship between income and pollution should be addressed to investigate multidimensional paths of relevant variables. Our paper is aimed at providing a first contribution in this direction.

\section{Acknowledgments}

The author is very grateful to Vania Paccagnan, Efrem Castelnuovo, Prof. J. Hutton and Prof. Riccardo Scarpa for their useful suggestions and comments. A special acknowledgment to Dr. Reyer Gerlagh for discussions about a previous version of this paper. He thanks prof. Charles Perrings and the other University of York (Department of Environment) participants of the workshop "Different uses of climate change models: preliminary results about the Environmental Kuznets Curve", York, March 2005, for valuable comments on a previous version of this paper. He thanks the ECINEQ (First Meeting of the Society for the study of Economic Inequality, Palma de Maiorca, 20 July 2005) conference participants for their valuable comments. Finally he thanks University of York workshop participants, York, December 2005 for useful suggestions.

\section{References}

Ansategui A. and Escapa M. (2002), "Economic growth and greenhouse gas emissions", Ecological Economics 40 23-37.

Arrow K. (1950), "A difficulty in the concept of social welfare”, Journal of Political Economy, 58, 328-346.

Atkinson A. B.,(1970), "On the measurement of inequality“, Journal of Economic Theory, vol.2, pp 244-263.

Atkinson A.B. and Bourgignon F. (1987), "Income distribution and differences in needs", Chapter 12 in G.R. Feiwel (ed.) Arrow and the Foundations of the Theory of Economic Policy, London: Mc Millan.

Azar C., Sterner T., (1996), "Discounting and distributional considerations in the context of global warming“, Ecological Economics, 19: 169-184.

Barbier (1997), "Introduction to the Environmental Kuznets Curve special issue", Environment and Development Economics, 2 (4), 369-381.

Bernstein P., Montgomery D., Rutheford T.(1999), "Global impacts of the Kyoto agreement: results from the MS-MRT model”, Resource and Energy Economics, 21, 375-413.

Bosello F., Roson F., (1998), "Carbon emissions trading and equity in international agreements", Nota di Lavoro FEEM 57/99.

Bosello, F., Buchner B., Carraro C., Raggi D. (2001), "Can Equity enhance efficiency? Lessons from the Kyoto Protocol”, Nota di Lavoro FEEM 49/2001.

Buonanno P., Carraro C., Galeotti M. (2003), "Endogenous induced technical change and the costs of Kyoto", Resource and Energy Economics 25 (2003), 11-34. 
Dagoumas A.S., Papagiannis G.K., Dokopoulos P.S. (2005), "An economic assessment of the Kyoto Protocol application”, Energy Policy, 34, 26-39.

Dardanoni V., Lambert P., (1988), "Welfare rankings of income distributions: a role for the variance and some insights for tax reform", Social Choice and Welfare, vol.5 pp1-17.

Davidson D., (1986), "Judging interpersonal interests", in Jon Elster and AAnund Hylland eds, Foundations of social choice theory. Cambridge: Cambridge University Press pp. 195-211.

Dinda S. (2004), "Environmental Kuznets Curve hpothesis: a survey", Ecological Economics, 49 (2004), 431-455.

Edenhofer O., Bauer N., Kriegler E. (2005), "The impact of technological change on climate protection and welfare: insights from the model MIND”, Ecological Economics, in press.

England R. (1998), "Measurement of social well-being; alternatives to gross domestic product", Ecological Economics, 25, 89-103.

Galeotti M., Buchner B. (2003), "Climate policy and Economic Growth in developing countries", Nota di Lavoro FEEM 95/2003.

Gowdy J., (2005), "Toward a New Welfare Economic for sustainability", Ecological Economics, $53,211-222$.

Jakeman G., Hanslow K., Hinchy M., Fisher B., Woffenden K. (2004),'Induced innovations and climate change policy", Energy Economics, 26, 937-960.

Kahneman D., Wakker P., Sarin R., (1997), "Back to Bentham? Explorations of experienced utility", The Quarterly Journal of Economics, vol.112, issue 2 pp. 375.

Kemfert C., (2005), "Induced technological change in a multi-regional, multi-sectoral integrated assessment model (WIAGEM). Impact assessment of climate policy strategies", Ecological Economics, 54, 293-305.

Kuik O., Gerlagh R. (2003), “Trade liberalisation and carbon leakage”, Energy Journal, 24 n.3.

Manne A.S, Mendehlson R.O., Richels R.G., (1995), "MERGE: a model for evaluating regional and global effects of GHG reduction policies", Energy policy, 23 (1): 17-34.

Nordhaus WD, (1991), "To slow or not to slow: the economics of the Greenhouse effect. Econ J. 101: 920-937.

Nordhaus (1994), "Managing the global commons", the Economics of climate change. MIT Press, Cambridge, Massachussetts.

Nordhaus, W.D. and Z. Yang (1996), “A Regional Dynamic General-Equilibrium Model of Alternative Climate-Change Strategies", American Economic Review, 4: 741-765.

Nordhaus W., Boyer J. (1999) "Rolling the DICE again: economic models of global warming", MIT Press, Cambridge, Massachussetts. 
$\mathrm{Ng}$ Y. (1999), "Utility, informed preference or happiness: following Harsany's argument to its logical conclusions", Soc. Choice and Welfare, 16, 197-216.

Panayatou T. (1993), "Empirical tests and policy analysis of environmental degradation at different stages of economic development", Working paper WP 238, Technology and Employment Programme, (Geneva, International Labour Office, 1993).

Rose A., (1992), "Equity consideration of tradable carbon entitlements", in S. Barrett et al., Combating global warming.

Rose A., Stevens Brandt, (1993), "The efficiency and equity of marketable permits for $\mathrm{CO}_{2}$ emissions", Resource and Energy Economics, 15: 117-146.

Selden T.M. and Song D. (1994), "Environmental quality and development: is there a Kuznets Curve for air pollution?", Journal of Environmental Economics and Management, vol. 27, pp.147162.

Sen, A. (1974), "Information Bases of Alternative Welfare Approaches: Aggregation and Income Distribution.” Journal of Public Economics 3, no. 4: 387-403.

Sen, A. (1976), “Real National Income.” Review of Economic Studies 43, no. 1: 19-39.

Sen A. (1999), “The possibility of social choice”, The American Economic Review, 89, 3, 349-378.

Shorrocks A., (1983), “Ranking income distributions”, Economica, vol.50, pp 1-17.

Stern D.I, Common M.S., Barbier E.B. (1996), "Economic growth and environmental degradation: a critique of the Environmental Kuznets Curve", World Development 24, 1151-1160.

Tol R., (2000), "Equitable cost-benefit analysis of climate change policies", Ecological Economics, 36: $71-85$.

WCED, (1987), "Our common future”, Oxford University Press and United Nations, New York. 